\title{
INFLUÊNCIA DO COMPORTAMENTO GREGÁRIO NOS PROCESSOS DE MUDA DE Rhipicephalus sanguineus (LATREILLE, 1806) (ACARI: IXODIDAE), EM CONDIÇÕES LABORATORIAIS*
}

\author{
PAULO HENRIQUE D. CANÇADO $^{1}$; ELIANE M. PIRANDA ${ }^{1}$; JOÃO LUIZ H. FACCINI ${ }^{2}$
}

\begin{abstract}
CANÇADO, P.H.D.; PIRANDA, E.M.; FACCINI, J.L.H. [Influence of aggregation on the molting process of Rhipicephalus sanguineus, (Latreille, 1806) (Acari: Ixodidae) under laboratory conditions.] Influência do comportamento gregário nos processos de muda de Rhipicephalus sanguineus (latreille, 1806) (acari: ixodidae), em condições laboratoriais. Revista Brasileira de Parasitologia Veterinária, v. 16, n. 4, p. 205-210, 2007. Curso de Pós-graduação em Ciências Veterinárias, Instituto de Veterinária, Universidade Federal Rural do Rio de Janeiro, Km 07 da BR 465, Seropédica, RJ, 23890-000. E-mail: cancadop@bol.com.br

The objective of this experiment was to evaluate the influence of different aggregations on the molting process of Rhipicephalus sanguineus (Latreille, 1806). Tick-naïve domestic rabbits (Oryctolagus cuniculus) were used as experimental hosts for the parasitic phase. A total of 10 rabbits were used on the experiment. All tick free-living phases were kept in incubator at $27 \pm 1^{\circ} \mathrm{C}, 80 \pm 5 \%$ relative humidity and scotophase. The periods of pre-molt, molt, percentage of ecdisis and molt rhythm of larvae and nymphs were studied. A total of 1560 engorged larvae and 1560 nymphs of $R$. sanguineus were divided in six groups of aggregations: 1, 5, 10, 20, 40 and 80 ticks with ten repetitions each one. The ecdises of nymphs and adults were daily observed. The percentage of ecdysis was the only parameter with no aggregations effects. All others parameters were statistically different $(p<0,05)$. The results suggested that highest aggregations accelerate the molting process.
\end{abstract}

KEY WORDS: Molt, aggregation, Ixodidae.

\section{RESUMO}

Objetivou-se com o experimento verificar a influência de diferentes agregações sobre os parâmetros relacionados ao processo de ecdise ninfal e de adulto de Rhipicephalus sanguineus (Latreille, 1806). Foram utilizados como hospedeiros 10 coelhos domésticos (Oryctolagus cuniculus) primos-infestados. Foram analisados os períodos de pré-ecdise, de ecdise e de muda, o percentual e o ritmo de ecdise para todos os estágios estudados. Durante toda a fase não-parasitária, os carrapatos foram mantidos em condições controladas de umidade $(80 \pm 5 \%)$ e temperatura $\left(27 \pm 1^{\circ} \mathrm{C}\right)$. Um total de 1560 larvas ingurgitadas e de 1560 ninfas ingurgitadas foi utilizado. Os carrapatos foram divididos em seis grupos contendo um, cin-

*Sob os auspícios do CNPq e CAPES/PROEX.

${ }^{1}$ Curso de Pós-Graduação em Ciências Veterinárias, Instituto de Veterinária (IV), Universidade Federal Rural do Rio de janeiro (UFRRJ), Km 7 da BR 465, Seropédica, RJ 23890-000, Brasil. E-mail: cancadop@bol.com.br

${ }^{2}$ Departamento de Parasitologia Animal/ IV, UFRRJ, Km 7 da BR 465, Seropédica, RJ 23890-000. co, dez, 20, 40 e 80 espécimes cada. Para cada um desses grupos foram realizadas dez repetições. Os processos de ecdise ninfal e de adulto foram acompanhados diariamente. Após a análise dos resultados, verificou-se que não houve relação entre as diferentes agregações e o percentual de ecdise em nenhuma etapa experimental. Em todos os outros parâmetros biológicos estudados, verificaram-se diferenças significativas $(p<0,05)$. Os resultados obtidos sugerem que em elevadas agregações o processo de ecdise tende a ser mais rápido.

PALAVRAS-CHAVE: Muda, agregação, Ixodidae.

\section{INTRODUÇÃO}

O processo de estabelecimento e manutenção de colônias de laboratório de artrópodes depende de vários fatores, incluindo equipamento adequado e pessoal qualificado no manuseio e conhecimento biológico da espécie que se pretende criar/ manter em laboratório. Neste sentido, a relação entre número de carrapatos e o espaço disponível é um dos fatores que podem determinar a velocidade do ciclo biológico, assim, este é um dos importantes parâmetros que devem ser 
considerados para o sucesso da colônia. Em estudo sobre o comportamento gregário de Haemaphysalis leporispalustris, Cançado et al. (2007) observaram, além de outros fatores, uma relação entre o aumento da agregação e o ritmo de ecdise.

Em um artigo de revisão, Zuben (2000) reuniu algumas referências indicando que a agregação pode aumentar a competição por espaço, com conseqüências deletérias para a população. Em contrapartida, esta mesma agregação poderia contrabalançar os efeitos deletérios pela maior sobrevivência do grupo, através do aumento da capacidade defensiva ou modificações do micro-clima e micro-habitat.

Rhipicephalus sanguineus (Latreille, 1806) é uma das espécies que possuem o comportamento gregário de forma marcante, cujos indivíduos são freqüentemente encontrados agrupados em frestas ou fendas, formando verdadeiros "ninhos de carrapatos”. É de reconhecida importância na transmissão de agentes causadores de Erlichiose e Babesiose Canina (SHAW et al., 2001) e, nos últimos anos, vem sendo investigada pelo seu papel na epidemiologia da Leishmaniose Visceral Canina (COUTINHO et al., 2005). Perfeitamente adaptada ao ambiente urbano, esta espécie está relacionada ao domicílio, em íntimo contato com humanos. Apesar da elevada especificidade para canídeos, já existem relatos de parasitismo em humanos (ESTRADA-PEÑA, JONGEJAN, 1999; DANTAS-TORRES et al., 2005). Infecções naturais de patógenos, como Rickettsia rickettsii e Bartonella henselae já foram registradas (BUSTAMANTE, 1943; DEMMA et al. 2005; WIKSWO et al., 2007), tornado $R$. sanguineus potencial transmissora para o homem.

O objetivo deste trabalho foi estudar os efeitos do comportamento gregário nos estágios imaturos de $R$. sanguineus durante o processo de muda. E desta maneira entender melhor o processo de ecdise para facilitar a criação em condições laboratoriais.

\section{MATERIAL E MÉTODOS}

O experimento foi realizado no Laboratório de Ixodologia do Departamento de Parasitologia Animal (DPA), Instituto de Veterinária (IV), localizado na Estação para Pesquisas Parasitológicas W. O. Neitz, da Universidade Federal Rural do Rio de Janeiro, Município de Seropédica, RJ, Brasil.

Como hospedeiros foram utilizados coelhos domésticos (Oryctolagus cuniculus) mestiços (Nova Zelândia X Califórnia), de ambos os sexos, cedidos a título de empréstimo pelo Setor de Cunicultura do Instituto de Zootecnia/ UFRRJ. Estes foram mantidos em gaiolas individuais, alimentados com ração comercial para coelhos e água fornecida $a d$ libitum. Neste experimento, foi seguido o protocolo de Princípios Éticos em Pesquisa Animal adotado pelo Colégio Brasileiro de Experimentação Animal (COBEA).

Os carrapatos utilizados no experimento foram oriundos da segunda geração de uma colônia pré-estabelecida no Laboratório de ixodologia / UFRRJ - DPA/IV, mantida em condições controladas de temperatura e umidade relativa (UR). Durante todo o experimento a fase não-parasitária foi mantida em estufa incubadora tipo B.O.D regulada a temperatura de $27 \pm 1^{\circ} \mathrm{C}$ e $80 \pm 5 \% \mathrm{UR}$.

Todas as infestações seguiram a técnica utilizada por Neitz et al. (1971), que consiste de um saco de orelha confeccionado com pano, pasta UNA e esparadrapo para sua fixação no coelho. Foram utilizados cinco coelhos para alimentação das larvas e outros cinco para as ninfas. Cada coelho recebeu aproximadamente 2500 larvas (12500 larvas para cada grama de ovos) com 25 dias de idade segundo as orientaçoes de Sartor (1994) e aproximadamente 1000 ninfas (3600 ninfas para cada grama de larvas ingurgitadas) com 25 dias de idade, baseando-se em Fernandes et al. (2004).

A partir do primeiro dia após a infestação, os sacos de pano foram abertos para limpeza e coleta dos espécimes ingurgitados, tão logo se iniciou o desprendimento. Todos os dias, as larvas ingurgitadas recuperadas foram limpas com pincel de cerdas macias. Parte do material foi utilizado para montagem do experimento e o restante foi novamente armazenado em seringas plásticas cortadas, fechadas com buchas de algodão e mantidas sob as mesmas condições de temperatura e umidade relativa já mencionadas.

Essas larvas ingurgitadas deram origem as ninfas que foram utilizadas para a segunda etapa do experimento. O processo de infestação e recuperação para obtenção das ninfas ingurgitadas foi o mesmo anteriormente descrito para larvas.

O delineamento experimental seguiu os moldes publicados por Cançado et al. (2007), ou seja, 1560 larvas ingurgitadas para ecdise ninfal e 1560 ninfas ingurgitadas para ecdise de adulto foram divididas em seis grupos A, B, C, D, E e F, contendo um, cinco, dez, 20, 40 e 80 espécimes cada respectivo grupo. Foram realizadas dez repetições por grupo. Os processos de ecdise ninfal e de adulto foram acompanhados diariamente.

Para a análise do período de pré-ecdise, percentual de ecdise e o período de muda, foram feitos testes de Análise de Variância (ANOVA) seguidos do teste de Tukey-Kramer. Quando, os desvios padrões mostraram diferença significativa entre as populações através do teste de Bartlett, foi necessária a realização do teste não paramétrico de Kruskal-Wallis seguido do teste de Dunn's. Quando foi analisado o período de ecdise, o grupo A foi retirado da análise por possuir apenas um indivíduo por frasco, não sendo possível realizar uma análise fidedigna com esses dados. Os demais grupos foram analisados pelos testes acima citados. A análise do ritmo de ecdise foi feita pela comparação das curvas de ecdise em gráficos de linhas. Os processos de muda para ninfa e adultos tiveram os mesmos parâmetros observados e analisados.

\section{RESULTADOS}

A análise dos resultados referentes ao processo de ecdise ninfal indicam que há diferenças $(\mathrm{p}<0,05)$ entre os tratamentos, conforme dispostos na Tabela 1 e Figura 1.

O período de pré-ecdise ninfal mostra uma clara tendência a diminuir quando se aumenta a agregação (Tabela 1). O limite mínimo foi alcançado pelo grupo E e repetido no grupo 
Tabela 1. Valores médios, seguidos de desvio padrão e limites, dos parâmetros relacionados à ecdise ninfal de Rhipicephalus sanguineus em seis níveis de agregação, mantidos em estufas tipo B.O.D. a $27 \pm 1^{\circ} \mathrm{C}$ e $80 \pm 5 \%$ UR.

\begin{tabular}{ccccccc}
\hline Parâmetros & \multicolumn{5}{c}{ Grupos com diferentes agregacões* } \\
\cline { 2 - 7 } Biológicos & $\mathrm{A}$ & $\mathrm{B}$ & $\mathrm{C}$ & $\mathrm{D}$ & $\mathrm{E}$ & $\mathrm{F}$ \\
& $\mathrm{n}=10$ & $\mathrm{n}=50$ & $\mathrm{~N}=100$ & $\mathrm{n}=200$ & $\mathrm{n}=400$ & $\mathrm{n}=800$ \\
\hline Pré-ecdise & $8,9 \pm 1,0^{\mathrm{a}}$ & $7,6 \pm 0,7^{\text {ab }}$ & $7,8 \pm 0,4^{\mathrm{ab}}$ & $7,3 \pm 0,7^{\mathrm{b}}$ & $7,0 \pm 0^{\mathrm{b}}$ & $7,0 \pm 0^{\mathrm{b}}$ \\
(dias) & $7-10$ & $7-9$ & $7-8$ & $6-8$ & $7-7$ & $7-7$ \\
Ecdise & - & $3,2 \pm 0,8^{\mathrm{b}}$ & $2,9 \pm 0,6^{\mathrm{c}}$ & $3,6 \pm 1,0^{\mathrm{c}}$ & $4,9 \pm 1,4^{\mathrm{a}}$ & $4,4 \pm 1,3^{\mathrm{b}}$ \\
(dias) & $\mathrm{c}-4$ & $2-4$ & $2-5$ & $3-7$ & $3-7$ & \\
Muda & $8,9 \pm 0,9^{\mathrm{a}}$ & $8,7 \pm 0,,^{7 \mathrm{bb}}$ & $8,6 \pm 0,7^{\mathrm{ac}}$ & $8,6 \pm 0,9^{\mathrm{ac}}$ & $8,7 \pm 1,8^{\mathrm{bc}}$ & $8,3 \pm 1,0^{\mathrm{c}}$ \\
(dias) & $7-10$ & $7-11$ & $7-10$ & $6-11$ & $7-20$ & $7-13$ \\
Percentual & $100 \pm 0 \mathrm{a}$ & $98 \pm 6,3^{\mathrm{a}}$ & $100 \pm 0^{\mathrm{a}}$ & $97,5 \pm 3,5^{\mathrm{ab}}$ & $98,2 \pm 3,4^{\mathrm{b}}$ & $97,8 \pm 2,9^{\mathrm{b}}$ \\
de ecdise & $100-100$ & $80-100$ & $100-100$ & $90-100$ & $90-100$ & $90-100$ \\
\hline
\end{tabular}

As letras A, B, C, D, E e F são os grupos com diferentes níveis de agregação $(1,5,10,20,40,80$ indivíduos/ frasco respectivamente) Médias seguidas de letras iguais, na mesma linha, quando estas não diferirem entre si $(p>0,05)$
F. Ambos os grupos obtiveram a mesma média, sete dias, e o desvio padrão foi zero. Em todos os grupos o valor mínimo foi alcançado, porém só nos grupos que tiveram maior agregação, as médias se igualaram aos limites inferiores.

As médias aqui obtidas foram significativamente diferentes: o grupo A obteve a maior média $(8,9 \pm 0,99$ dias) que foi diferente das médias dos grupos $\mathrm{E}$ e $\mathrm{F}$ (7,0 0 dias). O grupo $\mathrm{D}$ foi o único grupo em que o período de pré-ecdise atingiu o valor de seis dias (Tabela 1 ), e a sua média não diferiu significativamente dos grupos B, C, E e F.

O grupo E de $R$. sanguineus obteve a maior média para o

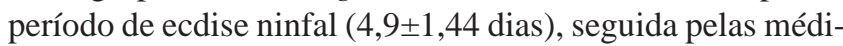
as dos grupos $\mathrm{F}, \mathrm{D}, \mathrm{B}$ e $\mathrm{C}$ respectivamente.

Para o período médio de muda ninfal observou-se reduçào gradativa, exceto para o grupo $\mathrm{E}$, na medida em que se aumentou o número de carrapatos, caracterizando redução do período não parasitário. O grupo E foi o único grupo que fugiu a regra, isto pode ser explicado pelo limite superior obser-
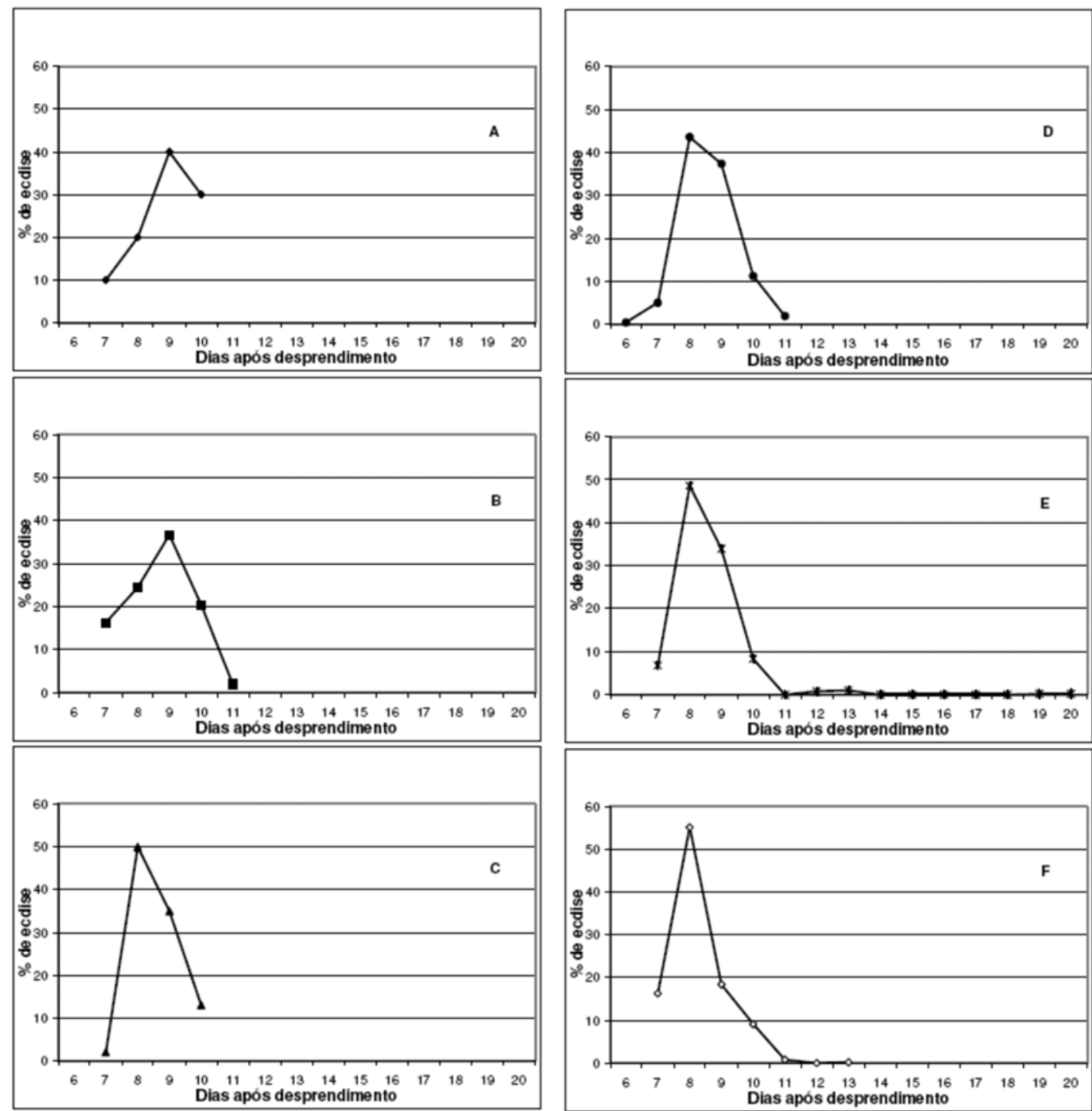

Figura 1. Ritmo de ecdise ninfal de Rhipicephalus sanguineus em diferentes níveis de agregação e a temperatura $\left(27 \pm 1^{\circ} \mathrm{C}\right)$ e umidade relativa $(80 \pm 5 \%)$ controladas. Grupo A: n=1; Grupo B: $n=5$; Grupo C: $n=10$; Grupo D: n=20; Grupo E: n=40; Grupo F: n=80. 
Tabela 2. Valores médios, seguidos de desvio padrão e limites, dos parâmetros relacionados à ecdise de adulto de Rhipicephalus sanguineus em seis níveis de agregação, mantidos em estufas tipo B.O.D. a $27 \pm 1^{\circ} \mathrm{C}$ e $80 \pm 5 \%$ UR.

\begin{tabular}{ccccccc}
\hline Parâmetros & \multicolumn{5}{c}{ Grupos com diferentes agregações* } \\
\cline { 2 - 7 } Biológicos & $\mathrm{A}$ & $\mathrm{B}$ & $\mathrm{C}$ & $\mathrm{D}$ & $\mathrm{E}$ & $\mathrm{F}$ \\
& $\mathrm{n}=10$ & $\mathrm{n}=50$ & $\mathrm{n}=100$ & $\mathrm{n}=200$ & $\mathrm{n}=400$ & $\mathrm{n}=800$ \\
\hline Pré-ecdise & $15,9 \pm 1,8^{\mathrm{a}}$ & $14,5 \pm 1,2^{\mathrm{ab}}$ & $13,1 \pm 0,7^{\mathrm{bc}}$ & $13,0 \pm 0,5^{\mathrm{bc}}$ & $12,8 \pm 0,6^{\mathrm{bc}}$ & $12,1 \pm 0,3^{\mathrm{c}}$ \\
(dias) & $14-19$ & $13-16$ & $12-14$ & $12-14$ & $12-14$ & $12-13$ \\
Ecdise & - & $6 \pm 2,7^{\mathrm{c}}$ & $8,2 \pm 2,0^{\mathrm{bc}}$ & $9,3 \pm 2,2^{\mathrm{b}}$ & $10,6 \pm 1,3^{\mathrm{ab}}$ & $12,4 \pm 1,8^{\mathrm{a}}$ \\
(dias) & $2-10$ & $5-11$ & $5-12$ & $8-12$ & $9-15$ & \\
Muda & $15,9 \pm 1,7^{\mathrm{ab}}$ & $16,8 \pm 2,4^{\mathrm{a}}$ & $15,8 \pm 2,4^{\mathrm{b}}$ & $15,9 \pm 2,4^{\mathrm{b}}$ & $15,9 \pm 2,4^{\mathrm{b}}$ & $16,1 \pm 2,8^{\mathrm{ab}}$ \\
(dias) & $12-19$ & $13-23$ & $12-24$ & $12-24$ & $12-24$ & $12-26$ \\
Percentual & $100 \pm 0^{\mathrm{a}}$ & $84 \pm 18,4^{\mathrm{a}}$ & $69 \pm 15,9^{\mathrm{b}}$ & $74 \pm 8,1^{\mathrm{b}}$ & $83 \pm 7,3^{\mathrm{ab}}$ & $83,5 \pm 5,4^{\mathrm{ab}}$ \\
de ecdise & $100-100$ & $60-100$ & $30-90$ & $60-90$ & $70-90$ & $77,5-93,75$ \\
\hline
\end{tabular}

As letras A, B, C, D, E e F são os grupos com diferentes níveis de agregação (1, 5, 10, 20, 40, 80 indivíduos/ frasco respectivamente)

Médias seguidas de letras iguais, na mesma linha, quando estas não diferirem entre si $(p>0,05)$
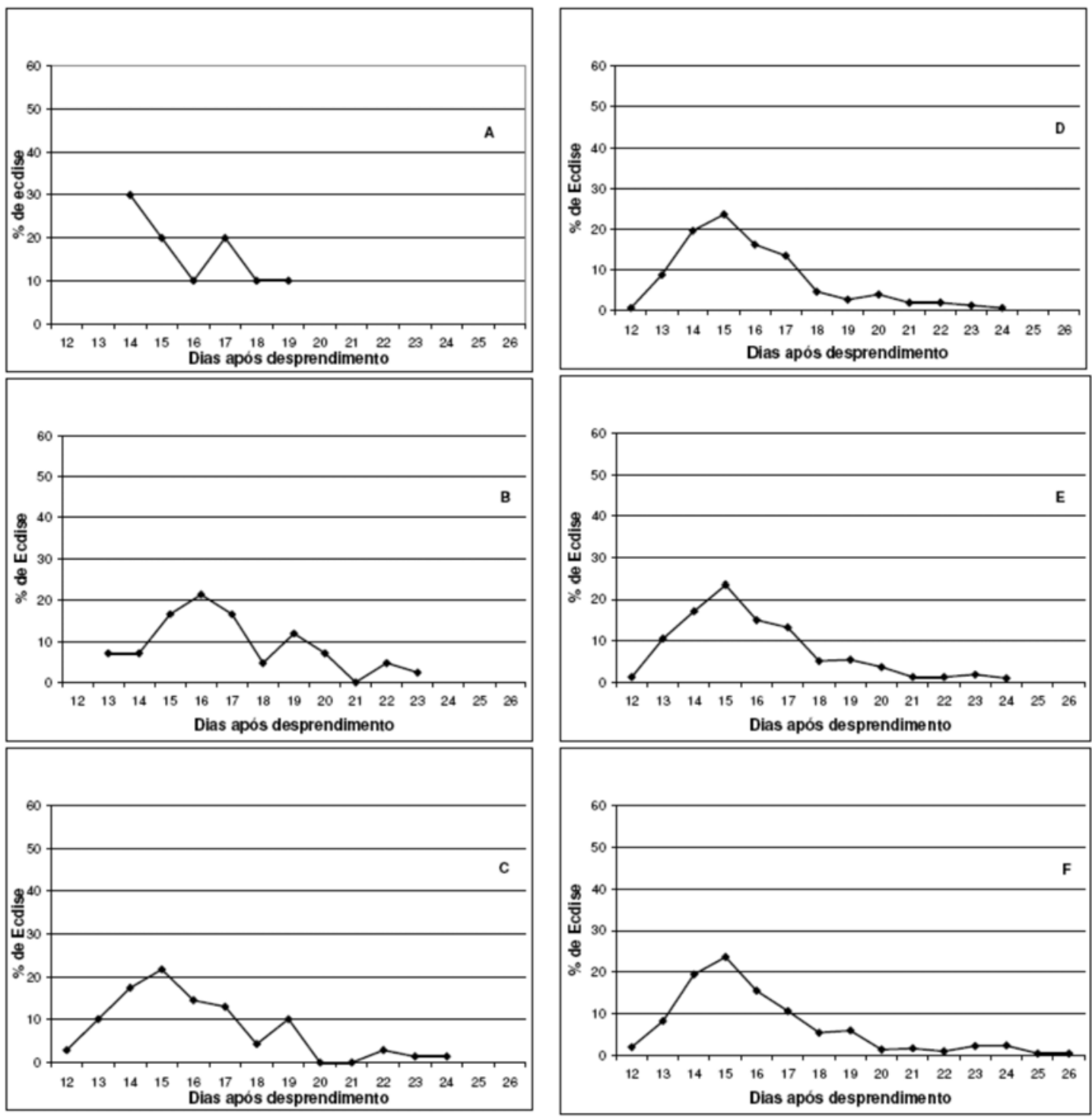

Figura 2. Ritmo de ecdise de adulto de Rhipicephalus sanguineus em diferentes níveis de agregação e a temperatura (27 $\left.\pm 1^{\circ} \mathrm{C}\right)$ e umidade

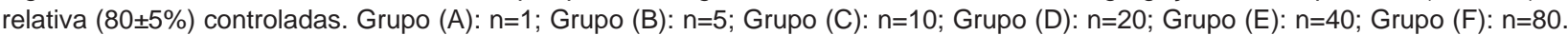


vado, de 20 dias. As médias obtidas para $R$. sanguineus foram significativamente distintas entre os seguintes grupos: o grupo A foi diferente dos grupos E e F. O grupo B também foi diferente do grupo $\mathrm{F}$.

Apesar de haver diferença nos valores médios do percentual de ecdise ninfal de $R$. sanguineus, esta diferença deve ser avaliada com cautela, já que todos os grupos obtiveram sucesso no processo de muda superior a $97,5 \%$. Os valores médios dos percentuais de ecdise ninfal variaram de $97,5 \pm 3,54$ (D) a $100,0 \pm 0$ (A e C).

Uma clara tendência à aceleração do ritmo de ecdise ninfal foi observada em grupos maiores. O dia modal ocorreu primeiro nos tratamentos onde os carrapatos foram mantidos agrupados. O grupo A, com apenas um indivíduo e o grupo B tiveram uma curva de ritmo distinta dos demais grupos, porém deve se levar em consideração o tamanho da amostra destes grupos (Figura 1).

É possível observar na Figura 1 que os grupos C, D, E e F tiveram o pico de ecdise bem marcado no oitavo dia após o desprendimento do hospedeiro. $\mathrm{O}$ grupo $\mathrm{F}$ foi o que teve o dia modal melhor definido com mais de $50 \%$ das ecdises ocorrendo no oitavo dia.

Os resultados referentes ao processo de ecdise de adulto indicaram que há diferenças entre os tratamentos $(p<0,05)$. Tais resultados estão dispostos na Tabela 2 e na Figura 2.

O periodo de pré ecdise do grupo A (15,9 1 1,79 dias) foi diferente dos grupos $\mathrm{C}(13,1 \pm 0,74$ dias), $\mathrm{D}(13 \pm 0,47$ dias), $\mathrm{E}$ (12,8 $\pm 0,63$ dias) e $\mathrm{F}(12,1 \pm 0,32$ dias) (Tabela 2$)$.

O período de muda não mostrou tendência de prolongamento ou de redução.

O percentual de ecdise de adulto foi particularmente distinto, porém sem que houvesse relação entre os valores obtidos e as diferentes agregações. Não existiu diferença significativa entre o grupo F e o grupo A. O grupo A $(100 \pm 0 \%)$ mostrou mais uma vez que a individualidade não é deletéria ao desenvolvimento. Os outros grupos tiveram as suas médias variando entre $69 \pm 15,95 \%$ (C) e $84 \pm 18,38 \%$ (B). Os resultados mostraram que a agregação não foi responsável pela mortalidade observada no processo de ecdise de adulto de $R$. sanguineus.

As curvas de ritmo de ecdise de adulto seguiram a tendência à redução do período não-parasitário. Para $R$. sanguineus, parece que pequenas agregações são suficientes para promover esta aceleração. Quase todos os grupos tiveram as curvas parecidas à exceção dos grupos $\mathrm{A}$ e $\mathrm{B}$ que foram distintos. $\mathrm{O}$ grupo $\mathrm{B}$ foi nitidamente mais lento e o grupo A teve o dia modal no primeiro dia de ecdise e outro pico de ecdises no quarto dia após o início (Figura 2).

\section{DISCUSSÃO}

Os resultados referentes ao período de pré-ecdise ninfal são semelhantes aos encontrados por Cançado et al. (2007) estudando $H$. leporispalustris, que mostrou redução do período quando se aumentou a agregação. Os resultados obtidos para o periodo de pré-ecdise de adulto estão em concordância com aqueles referentes ao processo de ecdise ninfal aqui relatados. Também ficou clara a tendência da diminuição dos valores médios do período de pré-ecdise quando se aumenta a agregação.

Olivieri e Serra-Freire (1984), estudando A. cajennese, consideraram que o período de ecdise é mais influenciado pelo tamanho da amostra do que por outros fatores. Para $H$. leporispalustris, Cançado et al. (2007) observaram que houve tendência a aumentar este período quando se aumentou a agregação e conseqüentemente o tamanho da amostra. Este achado não ficou evidente para ecdise ninfal de $R$. sanguineus (Tabela 1). Ainda sobre a ecdise ninfal, ocorreu uma redução no período de ecdise do grupo E para o grupo F. O mesmo pode ser observado na ecdise de adulto de $H$. leporispalustris (CANÇADO et al., 2007); entretanto este fenômeno não foi observado em $R$. sanguineus. É provável que utilizando-se níveis gregários ainda maiores oberve-se a redução do período de ecdise em agregações elevadas.

O período de muda é o parâmetro que melhor representa a velocidade do processo de ecdise (CANÇADO et al., 2007). Neste sentido ocorreu redução no periodo de muda ninfal em maiores agregações, e o periodo de muda de adulto não mostrou tendencia de prolongamento ou redução.

Wang et al. (2001), estudando a alimentação de forma agregada de $R$. appendiculatus, verificou aceleração do ingurgitamento e conseqüente redução do período parasitário em carrapatos que se alimentavam em grupo. $\mathrm{O}$ autor atribuiu este resultado à ação da saliva dos carrapatos que inibem o sistema imune do hospedeiro. Os carrapatos que não se alimentavam em grupo foram prejudicados pelo padrão de alimentação agregado. Quando os carrapatos agregados se desprendiam, promoviam uma resposta imune mais forte sobre aqueles que se alimentavam sozinhos. Diversos outros autores já constataram os benefícios da alimentação de forma agregada (SONENSHINE; ATWOOD, 1967; HAZLER; OSTFELD, 1995; RECHAV et al., 1997; LEVIN; FISH, 1998).

Haemaphysalis leporispalustris (CANÇADO et al., 2007) pareceu ser mais sensível às diferenças nas agregações quando comparado a $R$. sanguineus. Este por sua vez mostrou que baixas agregações são suficientes para provocar aceleração da ecdise ninfal. Quanto ao processo de ecdise de adulto de $R$. sanguineus, o padrão de aceleração do processo em função de maiores agregações não foi muito claro. Porém, a análise do gráfico de ritmo indica este fenômeno.

Não ocorreu prejuízo no percentual de ecdise em qualquer agregação utilizada. Entretanto, nas maiores agregações, 8,9 e 17,8 indivíduos/ml. (grupos E e F, respectivamente) a visualização do processo fica prejudicada em função dos resíduos resultantes do processo de muda. Desta forma, a utilização destas agregações para experimentos científicos não é aconselhada. Para a manutenção de colônias em laboratório estas podem ser utilizadas.

As agregações de até 4,4 indivíduos/ml (grupo D) propi- 
ciam uma boa visualização de todo o processo de ecdise sendo estas as agregações recomendadas para a experimentação científica. Quando necessária à manutenção de indivíduos isoladamente pode ser utilizada sem prejuízos para a colônia.

\section{REFERÊNCIAS BIBLIOGRÁFICAS}

BUSTAMANTE, M.E. Una nueva rickettsiosis en MexicoExistencia de La Fiebre Manchda Americana en los Estados de Sinaloa y Sonora. Revista Del Instituto de Salubridad y Enfermidades Tropicales, v. 4, n. 3, p. 189-211, 1943.

CANÇADO, P.H.D.; PIRANDA, E.M.; FACCINI, J.L.H.; DAEMON, E. Influência do comportamento gregário nos processos de muda de Haemaphysalis leporispalustris (Acari: Ixodidae), em condições laboratoriais. Revista Brasileira de Parasitologia Veterinária. v.16, n.1, p. 47-53, 2007

COUTINHO, M. T. Z. ; BUENO, L. L.; STERZIK, A.; FUJIWARA, R. T.; BOTELHO, J. R.; MARIA, M.; GENARO, O.; LINARDI, P. M.. Participation of Rhipicephalus sanguineus (Acari:Ixodidae) in the epidemiology of canine visceral leishmaniasis. Veterinary Parasitology, v. 128, n.1-2, p.149-155, 2005.

DANTAS-TORRES, F.; BRANDÃO-FILHO, S.P; FIGUEREDO, L.A.. Primeira descrição de parasitismo humano por Rhipicephalus sanguineus (Acari: Ixodidae) no Brasil. Revista da Sociedade Brasileira de Medicina Tropical, v.38, supl.1, p.411-415, 2005.

DEMMA, L.J.; TRAEGER, M.D.; NICHOLSON, W.L.; PADDOCK, C.D.; BLAU, D.M.; EREMEEVA, M.E.; DASCH, G.A.; LEVIN, M.L.; SINGLETON, J.; ZAKI, S.R.; CHEEK, J.E.; SWERDLOW, D.L.; MCQUISTON, J.H. Rocky Moutain spotted fever from an unexpected tick vector in Arizona. New England Journal of Medicine, v.353, n. 6, p. 587-594, 2005.

ESTRADA-PEÑA, A.; JONGEJAN, F. Ticks feeding on humans: a review of records on human-biting Ixodoidea with special reference to pathogen transmission. Experimental and Applied Acarology, v. 23, n. 9, p. 685-715, 1999.

FERNANDES, J.I.; CANÇADO, P.H.D.; PIRANDA, E.M.; FREITAS, L.H.T.; FACCINI, J. L. H. Relação entre peso e número de espécimes imaturos ingurgitados de Rhipicephalus sanguineus (Latreille, 1806) (Acari: Ixodidae) alimentados em coelhos domésticos. Revista
Brasileira de Parasitologia Veterinária, v. 13, supl.1, p. 321, 2004.

HAZLER, K.R.; OSTFELD, R.S. Larval density and feeding success of Ixodes scapularis on two species of Peromyscus. Journal of Parasitology, v.85, n. 6, p. 870-875, 1995.

LEVIN, M.L.; FISH, D. Density dependent factors regulating feeding success of Ixodes scapularis larvae (Acari; Ixodidae). Journal of Parasitology, v. 84, n. 1, p. 36-43, 1998.

NEITZ, W.O.; BOUGHTON, F.; WALTERS, H.S. Laboratory investigations on the karoo paralysis tick (Ixodes rubicundus Neumann, 1904). Onderstepoort Journal Veterinary Research, v. 38, n. 3, p. 215-224, 1971.

OLIVIERI, J.A.; SERRA-FREIRE, N.M.S. Estádio larval do ciclo biológico de Amblyomma cajennense. Arquivos da Universidade Federal Rural do Rio de Janeiro, v.7, n. 2, p.139-148, 1984.

RECHAV, Y.; GOLDBERG, M.; FIELDEN, L.J. Evidence for attachment pheromones in the Cayenne Tick (Acari: Ixodidae). Journal of Medical Entomology, v. 34, n. 2, p. 234-237, 1997.

SARTOR, A.A. Aspectos da biologia de Rhipicephalus sanguineus (Latreille, 1806) (Acarina: Ixodidae) em condições de laboratório. 1994. 80f. Tese (Doutorado) - Universidade Federal Rural do Rio de Janeiro, Itaguaí, 1994.

SHAW, S.E.; DAY, M.J.; BIRTLES, R.J.; BREITSCHWERDT; E.B. Tick-borne infectious diseases of dogs. Trends in Parasitology, v. 17, n. 2, p. 74-80, 2001.

SONENSHINE, D. E.; ATWOOD, E.L. Dynamics of feeding of the american dog tick, Dermacentor variabilis (Acarina: Ixodidae). Annals of the Entomological Society of America, v. 60, n.2, p. 362-372, 1967.

WANG, H.; HAILS, R.S.; CUI, W.W.; NUTTALL, P.A. Feeding aggregation of the Rhipicephalus appendiculatus (Ixodidae): benefits and costs in the contest with host response. Parasitology, v.123, n. 5, p. 447-453, 2001.

WIKSWO, M.E; HU, R.; METZGER, M.E.; EREMEEVA, M.E. Detection of Rickettsia rickettsii and Bartonella henselae in Rhipicephalus sanguineus Ticks from California. Journal of Medical Entomology, v. 44, n.1, p. 158-162, 2007.

ZUBEN, C.J.V. Implicações do fenômeno de agregação espacial para a dinâmica de populações de insetos. I-Competição por recursos alimentares e espaço. Revista Brasileira de Zoociências, v. 2, n. 1, p. 117-133, 2000.

Recebido em 17 de abril de 2007.

Aceito para publicação em 19 de outubro de 2007. 